\title{
WAVELENGTH DEPENDENT EFFECTIVE TRAP DENSITY IN CdTe : EVIDENCE FOR THE PRESENCE OF TWO PHOTOREFRACTIVE SPECIES
}

\author{
Ph. Delaye(1), L.A. de Montmorillon(1), I. Biaggio(1), J.C.Launay(2), G.Roosen(1)
}

(1) Institut d'Optique Théorique et Appliquée, Unité de Recherche Associée 14 au Centre National de la Recherche Scientifique, Bât.503, Centre Scientifique d'Orsay, B.P. 147, 91403 Orsay Cedex, France.

Tel: 33169416855, Fax: 33169413192, Email: gerald.roosen@iota.u-psud.fr

(2) 3-AR Action Aquitaine de Recherche en Apesanteur, B.P. 11, 33165 Saint Médard en Jalles Cedex, France and Institut de Chimie de la Matière Condensée de Bordeaux, Avenue du Docteur A.

Schweitzer, 33608 Pessac Cedex, France.

\begin{abstract}
:
An accurate determination of the effective trap density in CdTe:V is performed using both counterpropagating (small grating spacing) and copropagating (large grating spacing) two wave mixing. This enhanced precision allows to show a variation of the effective trap density with wavelength. We show that such a change is explained when two traps both coupled to the conduction and the valence bands are present. New theoretical expressions for the electron hole competition coefficient and Debye screening length are derived and used.
\end{abstract}


Photorefractive semiconductors like CdTe are characterized by a low dielectric constant compared to other photorefractive materials like sillenites or ferroelectrics. This point added to the fact that these materials are sensitive in the infrared region of the spectra leads to the point that even with a low effective trap density $\mathrm{N}_{\text {eff }}$ (around $10^{15} \mathrm{~cm}^{-3}$ ), the optimum grating vector (corresponding to $\mathrm{k}=\mathrm{k}_{0}$, with $\mathrm{k}_{0}$ the inverse of the Debye screening length) can not be obtained with copropagating beams. In other words, the photorefractive gain is mostly dominated by the diffusion field in most of the experiments. Then the use of curves of variation of the photorefractive gain with the grating spacing leads to a great uncertainty on the exact value of the effective trap density (compared to the good accuracy of the electron hole competition coefficient determined in these experiments). In order to decrease this uncertainty, we include in our measurement a value of two wave miwing gain obtained in a counterpropagating geometry. With this new experiment we not only deduce a more accurate value of the effective trap density, but also find that $\mathrm{N}_{\mathrm{eff}}$ is different for $1.06 \mu \mathrm{m}$ and $1.55 \mu \mathrm{m}$. In order to explain this wavelength dependence we use the photorefractive model with two photoactive traps developed by Bashaw [1], and show that this model can perfectly explain this variation of $\mathrm{N}_{\mathrm{eff}}$ with wavelength.

\section{Experimental study:}

The determination of the photorefractive parameters (electron-hole competition coefficient $\xi_{0}$ and effective trap density $\mathrm{N}_{\text {eff }}$ ) can be easily performed through a measurement of the photorefractive two beam coupling energy transfer gain $\Gamma$ as a function of the grating spacing $\Lambda$ (or as a function of the grating wave number $\mathrm{k}=2 \pi / \Lambda$ ) [2]. For s-polarized beams the expression of the gain according to the model with one defect coupled to both bands, when considering that the electron-hole competition factor is independent of the grating spacing, as usually admitted in semiconductors (diffusion length much longer than the grating spacing), is $[3,4]$ :

$$
\Gamma=\frac{2 \pi n_{0}^{3} r_{\text {eff }}}{\lambda \cos \theta} \frac{k_{B} T}{e} \frac{\xi_{0} k}{1+\frac{k^{2}}{k_{0}^{2}}}
$$

with $k_{0}^{2}=\frac{e^{2}}{\varepsilon k_{B} T} N_{e f f} . \theta$ is the half angle between the beams inside the crystal. The linear refractive index of the material is $\mathrm{n}_{0}, \mathrm{r}_{\text {eff }}$ is the effective electrooptic coefficient that depends on the crystal and the beam 
polarization orientations [5] and $\varepsilon$ is the dielectric constant. All these parameters are known from the literature.

In most of the cases, the experiments are performed with two beams which are copropagating with a small angle between them, leading to values of $\mathrm{k}$ that are smaller than $\mathrm{k}_{0}$. We can then very often work with a simplified expression :

$$
\Gamma=\frac{2 \pi n_{0}^{3} r_{e f f}}{\lambda \cos \theta} \frac{k_{B} T}{e} \xi_{0} k
$$

where the influence of $\mathrm{k}_{0}$ has disappeared. We can thus see that the experiments generally allow an accurate determination of the electron-hole coefficient $\xi_{0}$, whereas the $\mathrm{k}_{0}$ influence appears only through perturbations to the linear variation of $\Gamma$ with $\mathrm{k}$. Unless a great number of experimental points are used, the study of the variation of the photorefractive gain with the grating spacing gives only a poor estimation of the effective trap density present in the photorefractive material.

A solution is to have a measurement of the gain with a large value of $\mathrm{k}$. This is only possible in photorefractive semiconductors like CdTe by performing measurements with counterpropagating beams. These counterpropagating points generally lead to values of $\mathrm{k}$ such that we have $\mathrm{k}$ greater than $\mathrm{k}_{0}$, which allows to pass the maximum of the gain as a function of $\mathrm{k}$, giving a better determination of this maximum.

It is possible to get a feeling of the parameter accuracy that can be obtained from equation (1) and a given data set using a simple and general approach [6]. It consists of simulating a data set by using the theoretical function and a random number generator which imitates the known error distribution of each data point. Simulation of many data sets and many fits gives a statistically valid way to estimate the error of the parameters. Using function (1) with $\xi_{0}=1$ and $k_{0}^{2}=200$, a set of data points at $\mathrm{k}=1,1.5,2,2.5,3,4,5$, and assuming a gaussian $5 \%$ error distribution for each data point, one can generate a large number of data sets and fit Eq.(1) every time. From the distribution of $\xi_{0}, k_{0}^{2}$ values obtained over all the fits, one can estimate the confidence intervals for the parameters $\xi_{0}$ and $k_{0}^{2}$. The result for $90 \%$ confidence intervals is $\xi_{0}=[0.95,1.05]$ and $k_{0}^{2}=[100,900]$. Using a data set $\mathrm{k}=1,2,3,4,5,22$, which contains less data points but one point at large $\mathrm{k}$, the same procedure delivers, for the $90 \%$ confidence intervals, $\xi_{0}=[0.96,1.04]$ and $k_{0}^{2}=[174,230]$. In this example, the expected accuracy increase on the value of $k_{0}^{2}$ by the addition of a data point measured with counterpropagating beams, is evident. 
The above estimation, although mathematical, is very close to the experimental practice, as we will see now on an experimental example.

The CdTe:V sample we used, named B23-3, was already characterized through beam coupling measurements in the copropagating geometry [7]. The main results obtained in this geometry where : at $\lambda=1.06 \mu \mathrm{m}, 100<\mathrm{k}_{0}^{2}<300 \mu \mathrm{m}^{-2}$ (with a mean value of ${\overline{k_{0}}}^{2}=166 \mu \mathrm{m}^{-2}$ ), and $0.78<\xi_{0}<0.93$ (with a mean value of $\overline{\xi_{0}}=0.84$ ), at $\lambda=1.55 \mu \mathrm{m}, 50 \mu \mathrm{m}^{-2}<\mathrm{k}_{0}^{2}$ (with a mean value of $\overline{k_{0}}{ }^{2}=109 \mu \mathrm{m}^{-2}$ ), and $-0.94<\xi_{0}<-0.71$ (with a mean value of $\overline{\xi_{0}}=-0.82$ ).

at $\lambda=1.32 \mu \mathrm{m}$ the gain is zero which means that the electron-hole competition is strong, we have then $\xi_{0} \approx 0$ and $\overline{k_{0}} 2$ is indeterminate.

The measured sign of the electron hole competition says that the dominant carriers are electrons at $1.06 \mu \mathrm{m}$ and holes at $1.55 \mu \mathrm{m}$ [7]. We see on these results that the precision on the determination of $\xi_{0}$ is good whereas $\mathrm{k}_{0} 2$ is quite indeterminate.

To these results we can add transmission measurements that gives us the absorption of the crystal (Fig.1). Crystal absorption spectrum exhibits classical features with two absorption bands centered at $1 \mu \mathrm{m}$ and $1.5 \mu \mathrm{m}$ (Fig. 1). As for some of the other samples we studied, we see a background absorption $[7,8]$. This background absorption is assumed to be due to scattering centers and is supposed not to participate in the photorefractive effect. In the following, when saying that results are corrected from absorption background, we means that we consider the crystal absorption to be zero at $\lambda=2 \mu \mathrm{m}$. These corrected values of absorption are : $\alpha=1.6 \mathrm{~cm}^{-1}$ at $1.06 \mu \mathrm{m}, \alpha=0.85 \mathrm{~cm}^{-1}$ at $1.32 \mu \mathrm{m}$ and $\alpha=$ $0.58 \mathrm{~cm}^{-1}$ at $1.55 \mu \mathrm{m}$

One interest of the B23-3 crystal is its cut. Indeed, it is cut with a [111] polished face, (111) direction along the beam propagation direction whereas the other faces are [1 $\overline{1} 0]$ and $[11 \overline{2}]$, with the [1 $\overline{1} 0]$ plane as plane of incidence. In this configuration the effective electrooptic coefficient is non zero in both the co and counterpropagating geometries which facilitates the measurement. The effective electrooptic coefficient is $r_{\text {eff }}=\sqrt{\frac{2}{3}} r_{41}$ in the copropagating geometry and $r_{\text {eff }}=\sqrt{\frac{1}{3}} r_{41}$ in the counterpropagating geometry. With this configuration we can then easily add to our copropagating points, already presented, a counterpropagating point. Nevertheless if the absolute value of the gain is 
immediately obtained, care has to be taken in order to determine the sign of the gain compared to the copropagating case, i.e. to see if the majority carriers is the same at large and small grating spacings.

In order to measure the sign of the photorefractive gain we change the set-up to allow to go directly from one geometry to the other one changing the minimum of parameters (Fig.2). We choose an angle between the two beams of $90^{\circ}$ outside the crystal. A simple rotation of $90^{\circ}$ of the crystal allows us to go from a copropagating to a counterpropagating geometry. The crystal orientation is chosen with still [111] as the polished face, but with now the [11 $\overline{2}]$ plane as the plane of incidence. The value of the effective electrooptic coefficient is presented in Figure 3 for s polarizations. With spolarized beams and copropagating beams with $\theta_{\mathrm{c}}=0$, we have a gain that is zero. When turning a little the crystal, from this position, a small photorefractive gain with a certain sign appears (as $r_{\text {eff }}$ becomes different from zero), if we now continue to rotate the crystal until $\theta_{\mathrm{c}}=90^{\circ}$ (i.e. counterpropagating geometry) the sign of the effective electrooptic coefficient stays the same and so does the gain if the majority carrier for the photorefractive effect remains alike. Using this experiment at both wavelengths, at which gain was measured, we confirm that the sign of the majority carriers is the same whatever the grating spacing. We can now place the counterpropagating points on the experimental curves of variation of the photorefractive gain as a function of $\mathrm{k}$ (Fig. 4), after correction of the different value of the effective electrooptic coefficient in the two geometries.

We fit the data with the theoretical expression (1). These adjustments are conducted with the same value of $\mathrm{r}_{41}=5.5 \mathrm{pm} . \mathrm{V}^{-1}[9,10]$ for the two wavelengths and a refractive index $\mathrm{n}_{0}=2.82$ at $1.06 \mu \mathrm{m}$ and $\mathrm{n}_{0}=2.74$ at $1.55 \mu \mathrm{m}$ [11]. Using $r_{\text {eff }}=\sqrt{\frac{2}{3}} r_{41}$, we determine $\xi_{0}=0.80 \pm 0.03$ at $1.06 \mu \mathrm{m}$ and $\xi_{0}=-0.81 \pm 0.03$ at $1.55 \mu \mathrm{m}$ and $\mathrm{k}_{0}^{2}=262 \pm 30 \mu \mathrm{m}^{-2}$ at $1.06 \mu \mathrm{m}$ and $\mathrm{k}_{0}^{2}=96 \pm 13 \mu \mathrm{m}^{-2}$ at $1.55 \mu \mathrm{m}$. We see easily that the accuracy for $\mathrm{k}_{0}{ }^{2}$ is much better than the one determined with the copropagating points only. With the dielectric constant $\varepsilon=10.3$, one obtains an effective trap density $N_{\text {eff }}=(3.8 \pm 0.5) \times 10^{15} \mathrm{~cm}^{-3}$ at $1.06 \mu \mathrm{m}$ and $N_{\text {eff }}=(1.4 \pm 0.2) \times 10^{15} \mathrm{~cm}^{-3}$ at $1.55 \mu \mathrm{m}$.

Due to the fact that the counterpropagating point brings us an higher precision in the determination of the effective trap density we can note on these results that the effective trap density varies with the wavelength. Although this fact had already been observed in $\mathrm{BaTiO}_{3}$ crystals [12] it has never been really explained. We will now give indications for a possible origin of this variation of the effective trap density with wavelength. 


\section{The two traps model}

The model for the photorefractive effect had several evolutions since the model developed by Kuhktarev [3] that takes into account a single defect coupled to only one of the transport bands. It had rapidly been necessary to take into account two types of carriers [4] to explain some of the experimental results. Again, new results that does not fit the theory force to add to the model with one defect coupled with the two bands another defect coupled to one of the bands $[13,14]$.

One major characteristics of all these models is that with the assumption that the diffusion length of the carriers is much longer than the grating spacing (what is generally true in semiconductors) the expression of the gain can always be reduced to an expression like equation (1) with only the expression of $\xi_{0}$ and $k_{0}^{2}$ that varies from one model to another [3, 4, 13, 14]. Moreover, if we look at the different models in detail we can see that in all these models (in the limit of low illuminations, which corresponds to the regime generally used in experiments in semiconductors) the different concentrations of the defect species are constant with the wavelength and, more important, the value of $k_{0}^{2}$ is the same whatever the wavelength used. It means that none of these photorefractive models can explain our experimental results that show clearly such a variation.

Recently a new evolution of the photorefractive model appears [1], it takes into account two deep defects both coupled to both conduction and valence bands. The model established analytical expressions for the space charge field and showed that wavelength dependent charge redistribution between the two defects was possible. Such a charge redistribution can explain the variation of the effective trap density we observed.

Before looking at this, we simplify the expressions derived in Ref.[1]. First we limit ourselves to the case of the variation of the steady state space charge field with the grating spacing in the case where no external electric field is applied. Secondly we rewrite the expression found in Ref.[1], using the approximation of large diffusion lengths classically used in semiconductors, to show that the expression of the gain can be reduced to an expression of the form of equation (1) with new expressions for $\xi_{0}$ and $k_{0}^{2}$ that we derive. In every step of the calculations we compare the numerical results obtained with our 
expressions to the ones obtained without approximation with the general expressions of reference [1] (after correction of a typographical errors in formula (45) of Ref.[1]) to validate our expressions and our results.

\section{Derivation of the expressions}

We consider two traps (Fig.5), with total concentrations $\mathrm{N}_{\mathrm{T}}$ and $\mathrm{M}_{\mathrm{T}}$, present under two states of charges with ionized parts $\mathrm{N}$ and $\mathrm{M}$ and neutral parts $\mathrm{N}_{\mathrm{T}}-\mathrm{N}$ and $\mathrm{M}_{\mathrm{T}}-\mathrm{M}$ respectively. At each state we associate parameters for optical (S) and thermal (B) generation of carriers and recombination $(\gamma)$ in the deep traps. The superscript $(\mathrm{N}$ and $\mathrm{M}$ ) deals with the two different traps, whereas the subscript ( $\mathrm{n}$ and $\mathrm{p}$ ) deals with the two kinds of carriers (for example, $S_{p}^{N}$ represents the hole photoionization cross-section from the ionized state of the $\mathrm{N}$ trap). The different material equations that allow the calculation of the space charge field are [1]:

$$
\begin{aligned}
& \frac{\partial N}{\partial t}=\left(\beta_{n}^{N}+S_{n}^{N} I\right)\left(N_{T}-N\right)-\gamma_{n}^{N} n N-\left(\beta_{p}^{N}+S_{p}^{N} I\right) N+\gamma_{p}^{N} p\left(N_{T}-N\right) \\
& \quad \frac{\partial M}{\partial t}=\left(\beta_{n}^{M}+S_{n}^{M} I\right)\left(M_{T}-M\right)-\gamma_{n}^{M} n M-\left(\beta_{p}^{M}+S_{p}^{M} I\right) M+\gamma_{p}^{M} p\left(M_{T}-M\right) \\
& j_{n}=e n \mu_{n} E+\mu_{n} k_{B} T \frac{\partial n}{\partial x} \\
& j_{p}=e p \mu_{p} E-\mu_{p} k_{B} T \frac{\partial p}{\partial x} \\
& \frac{\partial n}{\partial t}=\frac{1}{e} \frac{\partial j_{n}}{\partial x}+\left(\beta_{n}^{N}+S_{n}^{N} I\right)\left(N_{T}-N\right)-\gamma_{n}^{N} n N+\left(\beta_{n}^{M}+S_{n}^{M} I\right)\left(M_{T}-M\right)-\gamma_{n}^{M} n M \\
& \quad \frac{\partial p}{\partial t}=-\frac{1}{e} \frac{\partial j_{p}}{\partial x}+\left(\beta_{p}^{N}+S_{p}^{N} I\right) N-\gamma_{p}^{N} p\left(N_{T}-N\right)+\left(\beta_{p}^{M}+S_{p}^{M} I\right) M-\gamma_{p}^{M} p\left(M_{T}-M\right) \\
& \frac{\partial E}{\partial x}=-\frac{e}{\varepsilon}\left(\left(N_{T}-N\right)+\left(M_{T}-M\right)+\left(N_{D}-N_{A}\right)+n-p\right)
\end{aligned}
$$

Where $\mathrm{n}$ and $\mathrm{p}$ are the free carriers concentrations, $\mathrm{j}_{\mathrm{n}}$ and $\mathrm{j}_{\mathrm{p}}$ are the electron and hole current densities and $\mathrm{E}$ is the space charge field. $\mathrm{N}_{\mathrm{D}}$ and $\mathrm{N}_{\mathrm{A}}$ are the donnors and acceptors, which compensation gives the semi insulating nature of the material. We limit ourselves to a one dimension problem with an illumination I given by a sinusoidal illumination pattern $I=I_{0}\left(1+\operatorname{Re}\left(m e^{i k z}\right)\right)$ of grating wave number $\mathrm{k}$ and modulation $\mathrm{m}$. 
We solve these equations in steady state, without applied electric field, in a classical way by linearizing the different quantities [1,3,4], with a non modulated part (with subscript 0 ) and a space modulated component with the same grating spacing than the illumination (with subscript 1). The zeroth order is given in Appendix A and allows to calculate the non modulated concentration of the different species that will be used in the first order expressions. The resolution of the first order system equation in order to obtain the modulated space charge field is long and tedious but does not present any mathematical difficulties. Under the assumption that the free carrier grating concentrations are much smaller than the one of gratings in the deep traps $\left(n_{1}, p_{1} \ll<N_{1}, M_{1}\right)$, we obtain the expression for modulated space charge field $\mathrm{E}_{1}$ :

$$
E_{1}=-i\left(m k \frac{k_{B} T}{e}\right) \frac{N U M}{D E N O M}
$$

where :

$$
\begin{aligned}
N U M= & k^{2}\left[\left(\alpha_{n}^{N}-\alpha_{p}^{N}\right) Z^{M}+\left(\alpha_{n}^{M}-\alpha_{p}^{M}\right) Z^{N}\right]+\left(Z^{M}-Z^{N}\right)\left[\left(\alpha_{n}^{N} \kappa_{n}^{M^{2}}-\alpha_{n}^{M} \kappa_{n}^{N^{2}}\right)-\left(\alpha_{p}^{N} \kappa_{p}^{M^{2}}-\alpha_{p}^{M} \kappa_{p}^{N^{2}}\right)\right] \\
& -\kappa_{n}^{2}\left(\alpha_{p}^{N} Z^{M}+\alpha_{p}^{M} Z^{N}\right)+\kappa_{p}^{2}\left(\alpha_{n}^{N} Z^{M}+\alpha_{n}^{M} Z^{N}\right)+\frac{\varepsilon k_{B} T}{e^{2} I_{0}}\left(X_{n}^{M}-X_{n}^{N}\right)\left[\kappa_{n}^{M^{2}}\left(\alpha_{n}^{N}-\alpha_{p}^{N}\right)-\kappa_{n}^{N^{2}}\left(\alpha_{n}^{M}-\alpha_{p}^{M}\right)\right] \\
& +\frac{\varepsilon k_{B} T}{e^{2} I_{0}}\left(X_{p}^{M}-X_{p}^{N}\right)\left[\kappa_{p}^{M^{2}}\left(\alpha_{n}^{N}-\alpha_{p}^{N}\right)-\kappa_{p}^{N^{2}}\left(\alpha_{n}^{M}-\alpha_{p}^{M}\right)\right] \\
D E N O M & =\left(k^{2}+\kappa_{n}^{2}\right)\left(k^{2}+\kappa_{p}^{2}\right) Z^{M} Z^{N}+\left(k^{2}+\kappa_{p}^{2}\right)\left(\frac{\varepsilon k_{B} T}{e^{2} I_{0}}\right)\left[Z^{N} \kappa_{n}^{M^{2}} X_{n}^{M}+Z^{M} \kappa_{n}^{N 2} X_{n}^{N}\right] \\
& +\left(k^{2}+\kappa_{n}^{2}\right)\left(\frac{\varepsilon k_{B} T}{e^{2} I_{0}}\right)\left[Z^{N} \kappa_{p}^{M^{2}} X_{p}^{M}+Z^{M} \kappa_{p}^{N^{2}} X_{p}^{N}\right]+\left(\frac{\varepsilon k_{B} T}{e^{2} I_{0}}\right)^{2}\left(X_{n}^{N} X_{p}^{M}-X_{p}^{N} X_{n}^{M}\right)\left(\kappa_{n}^{N^{2}} \kappa_{p}^{M^{2}}-\kappa_{p}^{N^{2}} \kappa_{n}^{M^{2}}\right)
\end{aligned}
$$

With the following notations that are used classically in the space charge field expressions [4]:

$\alpha_{n}^{N}=S_{n}^{N}\left(N_{T}-N_{0}\right)$ and $\alpha_{p}^{N}=S_{p}^{N} N_{0}$

$k_{0 N}^{2}=\frac{e^{2}}{\varepsilon k_{B} T} \frac{N_{0}\left(N_{T}-N_{0}\right)}{N_{T}}$

$\kappa_{n}^{N^{2}}=\frac{e}{k_{B} T} \frac{\gamma_{n}^{N} N_{0}}{\mu_{n}}$ and $\kappa_{p}^{N^{2}}=\frac{e}{k_{B} T} \frac{\gamma_{p}^{N}\left(N_{T}-N_{0}\right)}{\mu_{p}}$

$A_{n}^{N}=\frac{\left(\beta_{n}^{N}+S_{n}^{N} I_{0}\right)}{S_{n}^{N} I_{0}}$ and $A_{p}^{N}=\frac{\left(\beta_{p}^{N}+S_{p}^{N} I_{0}\right)}{S_{p}^{N} I_{0}}$

and the same expressions for the M defect.

The model also uses specific parameters :

$X_{n}^{N}=\frac{n_{0} e \mu_{n}}{\varepsilon}-\left(\beta_{n}^{N}+S_{n}^{N} I_{0}\right)-\gamma_{n}^{N} n_{0}$ and $X_{p}^{N}=\frac{p_{0} e \mu_{p}}{\varepsilon}-\left(\beta_{p}^{N}+S_{p}^{N} I_{0}\right)-\gamma_{p}^{N} p_{0}$ 
$X_{n}^{M}=\frac{n_{0} e \mu_{n}}{\varepsilon}-\left(\beta_{n}^{M}+S_{n}^{M} I_{0}\right)-\gamma_{n}^{M} n_{0}$ and $X_{p}^{M}=\frac{p_{0} e \mu_{p}}{\varepsilon}-\left(\beta_{p}^{M}+S_{p}^{M} I_{0}\right)-\gamma_{p}^{M} p_{0}$

$\kappa_{n}{ }^{2}=\kappa_{n}^{N^{2}}+\kappa_{n}^{M^{2}}$ and $\kappa_{p}^{2}=\kappa_{p}^{N^{2}}+\kappa_{p}^{M^{2}}$

$Y_{n}=\frac{A_{n}^{N} \alpha_{n}^{N}+A_{n}^{M} \alpha_{n}^{M}}{\kappa_{n}^{2}}$ and $Y_{p}=\frac{A_{p}^{N} \alpha_{p}^{N}+A_{p}^{M} \alpha_{p}^{M}}{\kappa_{p}^{2}}$

$Z^{N}=\frac{A_{n}^{N} \alpha_{n}^{N}+\kappa_{p}^{N 2} Y_{p}}{k_{0 N}^{2}}$ and $Z^{M}=\frac{A_{n}^{M} \alpha_{n}^{M}+\kappa_{p}^{M 2} Y_{p}}{k_{0 M}^{2}}$

To these definitions we can add some relations between the parameters, relations that are given in Appendix B.

Even if not in a factorized form, we can nevertheless note that the space charge field (Eq.4) has the form found in previous models, i.e. the diffusion field $E_{d}=k \frac{k_{B} T}{e}$ multiplied by a rational function with a numerator that is a polynomial of degree 1 in $\mathrm{k}^{2}$ and a denominator that is a polynomial of degree 2 in $\mathrm{k}^{2}$.

In order to simplify this expression we will now make further approximations. The first one which is classical in semiconductor materials is that the Debye screening length is much smaller than the diffusion length, according to our notations it will correspond to $k_{0 N}^{2}, k_{0 M}^{2}>>\kappa_{n}{ }^{2}, \kappa_{p}{ }^{2}$. Under this approximation we have :

$$
X_{n}^{N}=X_{n}^{M}=\frac{e^{2} I_{0}}{\varepsilon k_{B} T} Y_{n} \text { and } X_{p}^{N}=X_{p}^{M}=\frac{e^{2} I_{0}}{\varepsilon k_{B} T} Y_{p}
$$

The second approximation is linked to the experimental set-up we use and to the range of grating spacings on which we make the experiments, we thus have $k^{2}>>\kappa_{n}{ }^{2}, \kappa_{p}{ }^{2}$, i.e. a grating spacing much smaller than the diffusion length of carriers.

Under all these approximations we can rewrite the photorefractive space charge field as :

$$
E_{1}=-i\left(m \frac{k_{B} T}{e}\right) \frac{\xi_{0} k}{1+\frac{k^{2}}{k_{0}^{2}}}
$$

with

$$
k_{0}^{2}=k_{0 N}^{2}\left(\frac{Y_{n} \kappa_{n}^{N 2}+Y_{p} \kappa_{p}^{N 2}}{A_{n}^{N} \alpha_{n}^{N}+Y_{p} \kappa_{p}^{N^{2}}}\right)+k_{0 M}^{2}\left(\frac{Y_{n} \kappa_{n}^{M 2}+Y_{p} \kappa_{p}^{M 2}}{A_{n}^{M} \alpha_{n}^{M}+Y_{p} \kappa_{p}^{M^{2}}}\right)
$$

and

$$
\xi_{0}=\frac{1}{k_{0}^{2}}\left[k_{0 N}^{2}\left(\frac{\alpha_{n}^{N}-\alpha_{p}^{N}}{A_{n}^{N} \alpha_{n}^{N}+Y_{p} \kappa_{p}^{N^{2}}}\right)+k_{0 M}^{2}\left(\frac{\alpha_{n}^{M}-\alpha_{p}^{M}}{A_{n}^{M} \alpha_{n}^{M}+Y_{p} \kappa_{p}^{M^{2}}}\right)\right]
$$


The photorefractive gain is then written in the form of the equation (1) with new expressions of $\xi_{0}$ and $k_{0}^{2}$. This explains why we could adjust our experimental results with equation (1) but found different values for $\mathrm{N}_{\text {eff }}$ when changing wavelength (see experimental part). We can always define an apparent "electron-hole" competition coefficient and an apparent Debye screening length, that will characterize the strength of the photorefractive effect. It can be easily shown from equations (6) that the results of the previous models $[4,13,14]$ can be deduced from this model taking the right values of generation and recombination parameters.

\section{Adjustment to the experimental results}

To see whether this two photoactive traps model explains our experimental results, we look for a set of parameters that will give us theoretical values of $\xi_{0}$ and $k_{0}^{2}$ equal to the ones we found experimentally. To these two photorefractive parameters, we add the absorption of the crystal $\alpha=\alpha_{n}^{N}+\alpha_{p}^{N}+\alpha_{n}^{M}+\alpha_{p}^{M}$. So we have six experimental parameters, corresponding to the two values of the wavelengths $1.06 \mu \mathrm{m}$ and $1.55 \mu \mathrm{m}$ (as well as for $1.32 \mu \mathrm{m}$ for $\xi_{0}$ and $\alpha$ ). The free parameters of the calculations are all the coefficients for photoionisation, thermal emission, recombination of carriers and concentration of the defects, with the condition that between $1.06 \mu \mathrm{m}$ and $1.55 \mu \mathrm{m}$ we only change the wavelength dependent coefficients, i.e. the photoionization cross-sections. Moreover as in our experiments we did not see, for our sample, any illumination dependent effect in the conditions of illumination used, i.e. from 1 to $100 \mathrm{~mW} . \mathrm{cm}^{-2}$, we will also verify that the parameters we find will not vary with illumination.

The set of parameters we find is shown in Table 1, it allows us to calculate exactly the experimental parameters, without any peculiar problem. The set of parameters we determined is certainly not unique as the adjustment is performed with eleven free parameters (we are in illumination conditions where the thermal emission is negligible and thermal emission coefficients are only taken small and non zero) which is a lot. Complementary experiments should be performed in order to have other informations on the characteristics of the two defects, this is not the goal of this study.

Here again we note that a complete calculation with the model of Bashaw [1] using the parameters we found, gives the same theoretical curves (in the range where the approximations we have done are justified, i.e. at large values of k). 
The two defects we have taken have thermal emission coefficients that are low, this implies that the defects are both close to the mid-gap where the Fermi level is located [14]. Thus these two defects are different from the one found responsible for the diminution of the photorefractive gain under AC field $[15,16]$. Indeed it was shown that the reduction of the gain under AC field is due to a defect with a thermal emission coefficient around $10^{3}-10^{4} \mathrm{~s}^{-1}$, very different from the one we found.

\section{Conclusion}

The measurements we performed on our CdTe:V sample show that the effective trap density was a wavelength dependent parameter in this sample. This new observation cannot be explained by the usual models of the photorefractive effect. Only a model that takes into account two traps coupled to the two bands allows charge redistribution that can explain this variation of the effective trap density with wavelength. Using the model developped by Bashaw [1], we derived an expression for the variation of the gain with grating spacing that has the same form than the one given by previous models. We establish the new expressions for the electron hole competition $\xi_{0}$ and the Debye screening length (inverse square root of $k_{0}^{2}$ ) with the two traps. Using these expressions we show that it is possible to explain our experimental results with this two defect model using a set of parameters that is plausible. At this point of the study it is impossible to identify these defects and even to determine their parameters. Other experiments should be performed to have a set of parameters that can be determined unambiguously.

These experiments, as well as other studies [17], shows the importance of the inclusion of a counter propagating point in the measurement of the photorefractive gain as a function of the grating spacing, as it brings a considerable improvement of the accuracy in the determination of the Debye screening length. This is not only true on the point of view of the material characterization, but also for the application of photorefractive crystals as this parameter is important for the value of the gain in the counterpropagating geometry or for experiments under applied electric field.

\section{$\underline{\text { Acknowledgment }}$}

Support for this work by DRET (grant \#94-063) is gratefully acknowleged. 


\section{Appendix A :}

Under the condition of small concentration of free carriers $\left(n_{0}, p_{0} \ll N_{D}-N_{A}, N_{T}-N_{0}, M_{T}-M_{0}\right)$ the different non spatially modulated concentrations are solution of a four equations system :

$$
\begin{aligned}
& N_{0}+M_{0}=N_{T}+M_{T}+N_{A}-N_{D} \\
& n_{0}=\frac{\left(\beta_{n}^{N}+S_{n}^{N} I_{0}\right)\left(N_{T}-N_{0}\right)+\left(\beta_{n}^{M}+S_{n}^{M} I_{0}\right)\left(M_{T}-M_{0}\right)}{\gamma_{n}^{N} N_{0}+\gamma_{n}^{M} M_{0}} \\
& p_{0}=\frac{\left(\beta_{p}^{N}+S_{p}^{N} I_{0}\right) N_{0}+\left(\beta_{p}^{M}+S_{p}^{M} I_{0}\right) M_{0}}{\gamma_{p}^{N}\left(N_{T}-N_{0}\right)+\gamma_{p}^{M}\left(M_{T}-M_{0}\right)} \\
& N_{0}=N_{T} \frac{\left(\beta_{n}^{N}+S_{n}^{N} I_{0}\right)+\gamma_{p}^{N} p_{0}}{\left(\beta_{n}^{N}+S_{n}^{N} I_{0}\right)+\gamma_{n}^{N} n_{0}+\left(\beta_{p}^{N}+S_{p}^{N} I_{0}\right)+\gamma_{p}^{N} p_{0}}
\end{aligned}
$$

The system can easily be reduced to third order equation that gives $\mathrm{N}_{0}$, which value gives then $\mathrm{n}_{0}, \mathrm{p}_{0}$ and $\mathrm{M}_{0}$. We have 3 solutions, but only one satisfies the conditions $0 \leq N_{0} \leq N_{T}, 0 \leq M_{0} \leq M_{T}, n_{0} \geq 0$ and $p_{0} \geq 0$. We numerically solve the system to have the non modulated concentrations that will be used for the first order solutions.

\section{Appendix B :}

With the zeroth order equations we write relations between the different parameters that appears in the expressions of the space charge field. We have :

$$
\begin{aligned}
& k_{0 N}^{2} Z^{N}=A_{n}^{N} \alpha_{n}^{N}+\kappa_{p}^{N^{2}} Y_{p}=A_{p}^{N} \alpha_{p}^{N}+\kappa_{n}^{N^{2}} Y_{n} \\
& k_{0 M}^{2} Z^{M}=A_{n}^{M} \alpha_{n}^{M}+\kappa_{p}^{M^{2}} Y_{p}=A_{p}^{M} \alpha_{p}^{M}+\kappa_{n}^{M^{2}} Y_{n} \\
& p_{0}=\frac{e I_{0}}{\mu_{p} k_{B} T} Y_{p} \\
& n_{0}=\frac{e I_{0}}{\mu_{n} k_{B} T} Y_{n}
\end{aligned}
$$

We can also rewrite :

$$
\begin{aligned}
& X_{n}^{N}=\frac{e^{2} I_{0}}{\varepsilon k_{B} T}\left\lceil Y_{n}\left(1-\frac{\kappa_{n}^{N^{2}}}{k_{0 N}^{2}} \frac{\left(N_{T}-N_{0}\right)}{N_{T}}\right)-\frac{A_{n}^{N} \alpha_{n}^{N}}{k_{0 N}^{2}} \frac{N_{0}}{N_{T}}\right\rceil \\
& X_{p}^{N}=\frac{e^{2} I_{0}}{\varepsilon k_{B} T}\left\lceil Y_{p}\left(1-\frac{\kappa_{p}^{N 2}}{k_{0 N}^{2}} \frac{N_{0}}{N_{T}}\right)-\frac{A_{p}^{N} \alpha_{p}^{N}}{k_{0 N}^{2}} \frac{\left(N_{T}-N_{0}\right)}{N_{T}}\right\rceil
\end{aligned}
$$

With the same relation for the defect M.

These relations are used to show that the expressions for $\xi_{0}$ and $k_{0}^{2}$ are symmetrical when exchanging $\mathrm{N}$ and $\mathrm{M}$ on one hand and $\mathrm{n}$ and $\mathrm{p}$ on the other hand, a point that is not evidently seen on the expressions. 


\section{$\underline{\text { References : }}$}

[1] M.C. Bashaw, M. Jeganathan, L. Hesselink. J. Opt Soc. Am. B 11, 1743 (1994).

[2] M.B. Klein, G.C.Valley. J.Appl. Phys. 57, 4901 (1985)

[3] N.V. Kukhtarev, V.B. Markov, S.G. Odulov, M.S. Soskin, V.L. Vinetskii. Ferroelectrics 22, 949 and 961 (1979).

[4] F.P. Strohkendl, J.M.C. Jonathan, R.W. Hellwarth. Opt. Lett. 11, 312 (1986).

[5] J.C. Fabre, J.M.C. Jonathan, G.Roosen. Optics Comm. 65, 257 (1988).

[6] W.H. Press, B.P. Flannery, S.A. Teukolsky, W.T. Vetterling. "Numerical Recipes - the Art of Scientific Computing”, Second Edition, Cambridge university Press Cambridge (1992).

[7] L.A. de Montmorillon, Ph. Delaye, J.C. Launay, G. Roosen. Opt. Materials, 4, 233 (1995)

[8] J. C. Launay, V. Mazoyer, M. Tapiero, J.P. Zielinger, Z. Guellil, Ph. Delaye and G.Roosen, Appl. Phys. A 55, 33 (1993)

[9] A. Partovi, J. Millerd, E.M. Garmire, M. Ziari, W.H. Steier, S.B. Trivedi, M.B. Klein. Appl. Phys. Lett. 57, 846, (1990).

[10] D.B. Chenault, R.A. Chipman, S.Y. Liu. Appl. Opt. 33, 7382, (1994).

[11] D.T.F. Marple. J.Appl. Phys. 35, 539 (1964)

[12] J. Zhang, S.X. Dou, H. Gao, Y Zhu, P Ye. Appl. Phys. Lett. 67, 458, (1995).

[13] P. Tayebati. J. Opt Soc. Am. B 9, 415 (1992).

[14] Ph. Delaye, H.J. von Bardeleben, G.Roosen, Appl. Phys. A 59, 357 (1994)

[15] J.Y. Moisan, N. Wolffer, O. Moine, P. Gravey, G. Martel, A. Aoudia, R. Repka, Y. Marfaing, R. Triboulet. J. Opt Soc. Am. B 11, 1655 (1994).

[16] I. Biaggio, G. Roosen. "Influence of shallow traps on the enhancement of the photorefractive grating amplitude by a high frequency alternating electric field : a probabilistic analysis". To be published in J. Opt Soc. Am. B. 13, ํ 10, Special issue on Photorefractive Materials (october 1996) [17] K. Shcherbin, A Shumeljuk, S. Odoulov, P. Fochuk, G. Brost. "Relaxation of photorefractive gratings in Cadmium Telluride crystals”. SPIE Vol. 2795, 236 (1996) 


\section{Figure Captions :}

Figure 1 : Room temperature absorption spectra of sample B23

Figure 2 : Configuration used for the comparison of the sign of the gain at large grating spacing (copropagating geometry, $\theta_{\mathrm{c}}=0^{\circ}$ ) and at small grating spacing (counterpropagating geometry, $\theta_{\mathrm{c}}=90^{\circ}$ ). The arrows indicates the directions of the grating wave vectors $\overrightarrow{\mathrm{k}}$ in the two geometries.

Figure 3 : Value of the effective electrooptic coefficient as a function of the angle of rotation of the crystal. The beams are s-polarised.

Figure 4 : Experimental photorefractive gain as a function of the grating spacing at wavelengths $\lambda=1.06 \mu \mathrm{m}(\boldsymbol{O})$ and $1.55 \mu \mathrm{m}(\boldsymbol{\Delta})$ for CdTe sample B23. Solid lines are the best theoretical adjustments. The dashed lines represent the adjustment with $\sigma=2 \sigma_{\min }$. The photorefractive gain at $1.32 \mu \mathrm{m}$ is nearly zero.

Figure 5 : Energy diagram with two defects in total concentrations $\mathrm{N}_{\mathrm{T}}$ and $\mathrm{M}_{\mathrm{T}}$ both coupled to both the valence and the conduction band.

\section{Table Caption :}

Parameter used for the adjustment of the experimental results with the theoretical expression. $\mathrm{N}_{0}$ and $\mathrm{M}_{0}$ are calculated with the zeroth order equations. 
Table

\begin{tabular}{|c|c|c|c|}
\hline$\lambda(\mu \mathrm{m})$ & 1.064 & 1.32 & 1.55 \\
\hline $\mathrm{N}_{\mathrm{T}}\left(\mathrm{cm}^{-3}\right)$ & \multicolumn{3}{|c|}{$10^{16}$} \\
\hline $\mathrm{M}_{\mathrm{T}}\left(\mathrm{cm}^{-3}\right)$ & \multicolumn{3}{|c|}{$1.2 \times 10^{16}$} \\
\hline $\mathrm{N}_{\mathrm{D}^{-}} \mathrm{N}_{\mathrm{A}}\left(\mathrm{cm}^{-3}\right)$ & \multicolumn{3}{|c|}{$1.2 \times 10^{16}$} \\
\hline $\mathrm{N}_{0}\left(\mathrm{~cm}^{-3}\right)$ & $7.65 \times 10^{15}$ & $8.23 \times 10^{15}$ & $9.19 \times 10^{15}$ \\
\hline $\mathrm{M}_{0}\left(\mathrm{~cm}^{-3}\right)$ & $2.35 \times 10^{15}$ & $1.67 \times 10^{15}$ & $8.12 \times 10^{14}$ \\
\hline$S_{n}^{N}\left(\mathrm{~cm}^{2}\right)$ & $1.11 \times 10^{-16}$ & $2.55 \times 10^{-17}$ & $1.03 \times 10^{-18}$ \\
\hline$S_{n}^{M}\left(\mathrm{~cm}^{2}\right)$ & $1.34 \times 10^{-16}$ & $2.26 \times 10^{-17}$ & $8.77 \times 10^{-19}$ \\
\hline$S_{p}^{N}\left(\mathrm{~cm}^{2}\right)$ & $3.70 \times 10^{-18}$ & $6.33 \times 10^{-17}$ & $5.91 \times 10^{-17}$ \\
\hline$S_{p}^{M}\left(\mathrm{~cm}^{2}\right)$ & $1.85 \times 10^{-17}$ & $2.58 \times 10^{-17}$ & $3.46 \times 10^{-17}$ \\
\hline$\gamma_{n}^{N}\left(\mathrm{~cm}^{3} \mathrm{~s}^{-1}\right)$ & \multicolumn{3}{|c|}{$1.79 \times 10^{-9}$} \\
\hline$\gamma_{p}^{N}\left(\mathrm{~cm}^{3} \mathrm{~s}^{-1}\right)$ & \multicolumn{3}{|c|}{$2.39 \times 10^{-8}$} \\
\hline$\gamma_{n}^{M}\left(\mathrm{~cm}^{3} \mathrm{~s}^{-1}\right)$ & \multicolumn{3}{|c|}{$3.92 \times 10^{-7}$} \\
\hline$\gamma_{p}^{M}\left(\mathrm{~cm}^{3} \mathrm{~s}^{-1}\right)$ & \multicolumn{3}{|c|}{$1.25 \times 10^{-9}$} \\
\hline$\beta_{n}^{N}\left(\mathrm{~s}^{-1}\right)$ & \multicolumn{3}{|c|}{0.00248} \\
\hline$\beta_{p}^{N}\left(\mathrm{~s}^{-1}\right)$ & \multicolumn{3}{|c|}{0.000182} \\
\hline$\beta_{n}^{M}\left(\mathrm{~s}^{-1}\right)$ & \multicolumn{3}{|c|}{0.00458} \\
\hline$\beta_{p}^{M}\left(\mathrm{~s}^{-1}\right)$ & \multicolumn{3}{|c|}{0.000144} \\
\hline$k_{0}^{2}\left(\mu \mathrm{m}^{-2}\right)$ & 260 & $\mathrm{NA}$ & 98 \\
\hline$\alpha\left(\mathrm{cm}^{-1}\right)$ & 1.64 & 0.85 & 0.58 \\
\hline$\xi_{0}$ & 0.79 & 0 & -0.81 \\
\hline
\end{tabular}




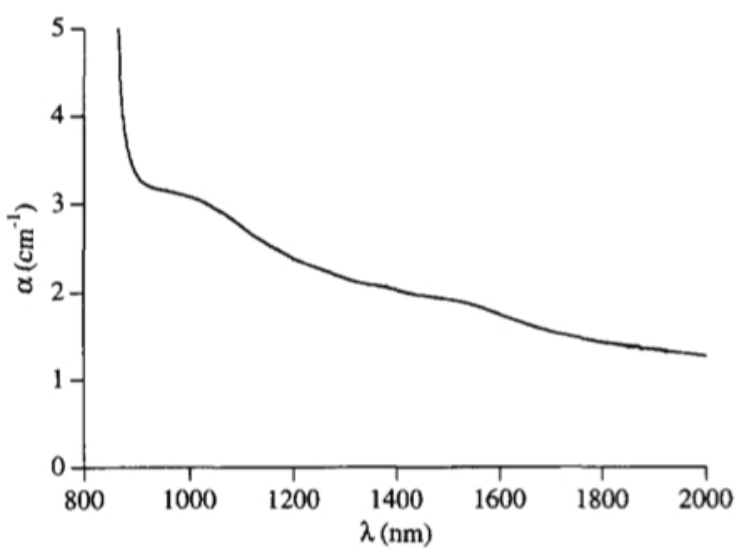

Figure 1
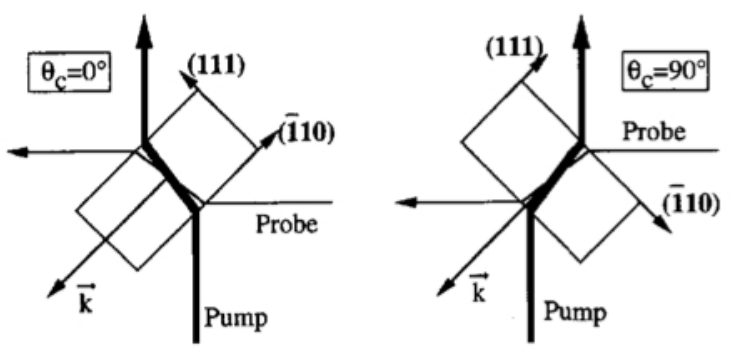

Figure 2

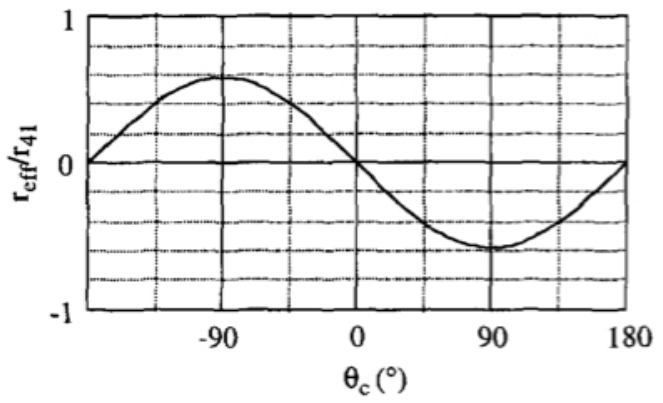

Figure 3

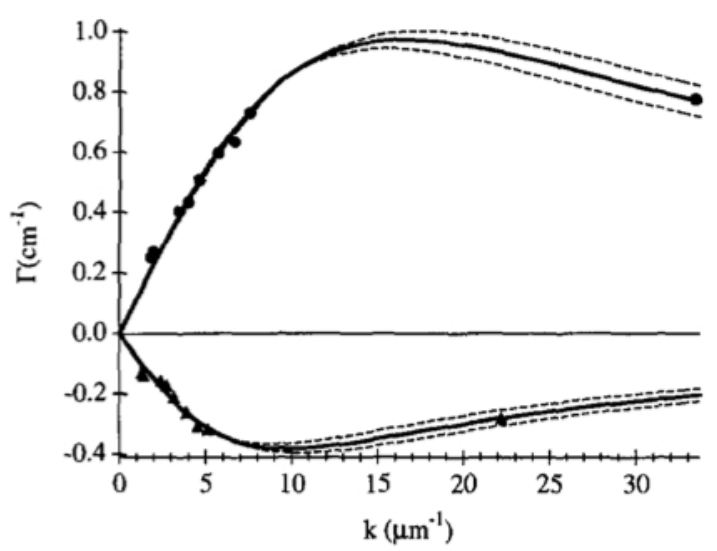

Figure 4 


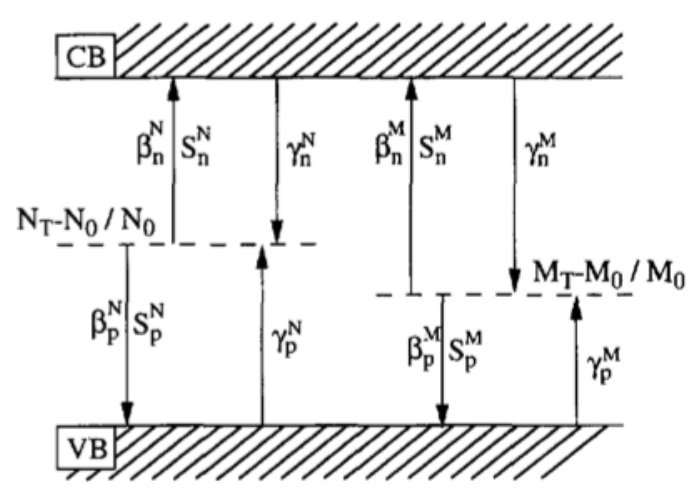

Figure 5 